\title{
Short-term cognitive changes after unilateral temporal lobectomy or unilateral amygdalo-hippocampectomy for the relief of temporal lobe epilepsy
}

\author{
Laura H Goldstein, Charles E Polkey
}

\begin{abstract}
Forty two patients who had unilateral temporal lobe surgery (either temporal lobectomy or amygdalo-hippocampectomy) were evaluated using a selection of cognitive tests before and soon after surgery, to examine whether the amygdalohippocampectomy produces less cognitive impairment than the standard en bloc resection. On specific indices of cognitive functioning an amygdalo-hippocampectomy rather than a temporal lobectomy, undertaken on the temporal lobe thought to mediate that particular function, produced less impairment, in terms of change in cognitive function resulting from the operation. An amygdalo-hippocampectomy carried out on the temporal lobe not thought to mediate such skills, however, resulted in less improvement or more deterioration in these functions than a temporal lobectomy, except in the case of delayed prose recall, where a right amygdalo-hippocampectomy led to more improvement than a right temporal lobectomy. Overall there were few scores which distinguished between the different surgical procedures for cognitive outcome.
\end{abstract}

(F Neurol Neurosurg Psychiatry 1993;56:135-140)

Neurosurgical treatment of temporal lobe epilepsy by unilateral anterior temporal lobectomy results in selective memory impairment. ${ }^{1-10}$ Verbal learning and memory impairments have been found following dominant temporal lobe resections and visuospatial learning and memory impairments following non-dominant temporal lobe resections. Where variable amounts of hippocampal tissue have been resected, different patterns of impairment, related to the size of the resection have been reported on some tasks. ${ }^{11}$

Short-term cognitive sequelae of unilateral Department of of Psychiatry, De Crespigny Park, London SE5 8AF, UK LH Goldstein

Maudsley Hospital, Denmark Hill, London SE5 8AZ, UK CE Polkey

Correspondence to: Dr Goldstein

Received 16 April 1991 and in revised form 27 November 1991.

Accepted 15 June 1992
The en bloc temporal lobectomy performed at the Maudsley Hospital ${ }^{14}$ involves the removal of between 5.5 and $6.5 \mathrm{~cm}$ of temporal lobe from the pole and includes the mesial temporal lobe structures. In dominant hemisphere resections only the anterior 1-2 cm of the superior temporal gyrus is removed. A less radical surgical intervention, selective amygdalo-hippocampectomy $(\mathrm{AH})^{15-17}$ has been described for patients in whom the epileptogenic focus can be localised to unilateral mesiobasal limbic structures. In this operation approximately 2-3 centimetres of hippocampus and a large part of the amygdala are removed together with the parahippocampal gyrus leaving less amygdala (a small portion of the antero-medial part) than in the standard en bloc procedure. Little comparative data on the cognitive sequelae of the two operations have been published. A report from Zurich compares six patients who had selective $\mathrm{AH}$ with five who had complete unilateral removal of the anterior two-thirds of the temporal lobe. ${ }^{17}$ A verbal memory deficit was found following left temporal lobectomy but not following left AH. Visual maze learning was impaired after right temporal lobectomy whereas the deficit was less after right $\mathrm{AH}$.

In view of the need to minimise cognitive deficits as well as achieve good seizure control by temporal lobe surgery a larger scale comparison of the two operations is required. Short-term cognitive outcome data will be presented on a preliminary series of 42 patients who have had either temporal lobectomy (TL) or AH since 1987 at the Maudsley Hospital.

\section{Method}

Subjects

We describe 42 patients who were right handed or had left hemisphere language dominance revealed during the intracarotid sodium amytal (Wada) procedure, who had temporal lobe surgery between 1987-90. All were aged above 16 years at time of surgery.

Characteristics are given in table 1 for the patients who had either a left or right temporal lobectomy (LTL or RTL) or a left or right amygdalo-hippocampectomy (LAH or RAH). Of the 42, 11 had RTL, 8 LTL, 9 the RAH operation and 14 the LAH operation.

Pre- and Postoperative investigations

All patients had a neuropsychologial assessment (see below), a CT scan and routine and 
Table 1 Patient characteristics for those having right or left amygdalo-hippocampectomy or temporal lobectomy and differences in mean values $(p<0.05)$

\begin{tabular}{|c|c|c|c|c|c|}
\hline $\begin{array}{l}N \\
\text { Sex }\end{array}$ & $\begin{array}{l}R T L \\
11 \\
5 F, 6 M\end{array}$ & $\begin{array}{l}L T L \\
8 \\
5 F, 3 M\end{array}$ & $\begin{array}{l}R A H \\
9 \\
6 F, 3 M\end{array}$ & $\begin{array}{l}L A H \\
14 \\
7 F, 7 M\end{array}$ & Difference \\
\hline $\begin{array}{l}\text { Age at surgery } \\
\text { (years-months) (AS) }\end{array}$ & $\begin{array}{l}32 \mathrm{yr} 1 \mathrm{~m} \\
(\mathrm{SD} 11 \mathrm{yr} 1 \mathrm{~m})\end{array}$ & $\begin{array}{l}28 \mathrm{yr} 10 \mathrm{~m} \\
(\mathrm{SD} 6 \mathrm{yr} 9 \mathrm{~m})\end{array}$ & $\begin{array}{l}29 \mathrm{yr} 5 \mathrm{~m} \\
(\mathrm{SD} 8 \mathrm{yr} 5 \mathrm{~m})\end{array}$ & $\begin{array}{l}25 \mathrm{yr} 9 \mathrm{~m} \\
(\mathrm{SD} 6 \mathrm{yr} 0 \mathrm{~m})\end{array}$ & NSig \\
\hline $\begin{array}{l}\text { Age at first seizure } \\
\text { (years-months) (AOS) }\end{array}$ & $\begin{array}{l}12 \mathrm{yr} 2 \mathrm{~m} \\
(\mathrm{SD} 7 \mathrm{yr} 9 \mathrm{~m})\end{array}$ & $\begin{array}{l}7 \mathrm{yr} 1 \mathrm{~m} \\
(\mathrm{SD} 5 \mathrm{yr} 7 \mathrm{~m})\end{array}$ & $\begin{array}{l}12 \mathrm{yr} 6 \mathrm{~m} \\
(\mathrm{SD} 10 \mathrm{yr} 11 \mathrm{~m})\end{array}$ & $\begin{array}{l}6 \mathrm{yr} 5 \mathrm{~m} \\
(\mathrm{SD} 6 \mathrm{yr} 8 \mathrm{~m})\end{array}$ & $\begin{array}{l}\text { left } \\
<\text { right }\end{array}$ \\
\hline $\begin{array}{l}\text { Age of onset of chronic epilepsy } \\
\text { (years-months) (AOCS) }\end{array}$ & $\begin{array}{l}14 \mathrm{yr} 2 \mathrm{~m} \\
(\mathrm{SD} 8 \mathrm{yr} 5 \mathrm{~m})\end{array}$ & $\begin{array}{l}8 \mathrm{yr} 9 \mathrm{~m} \\
(\mathrm{SD} 5 \mathrm{yr} 7 \mathrm{~m})\end{array}$ & $\begin{array}{l}12 \text { yr } 8 \mathrm{~m} \\
(\mathrm{SD} 10 \mathrm{yr} 9 \mathrm{~m})\end{array}$ & $\begin{array}{l}7 \mathrm{yr} 3 \mathrm{~m} \\
(\mathrm{SD} 7 \mathrm{yr} 6 \mathrm{~m})\end{array}$ & $\begin{array}{l}\text { left }< \\
\text { Right }\end{array}$ \\
\hline $\begin{array}{l}\text { Time between chronic onset } \\
\text { and surgery (years-months) (TCS) }\end{array}$ & $\begin{array}{l}18 \mathrm{yr} 4 \mathrm{~m} \\
(\mathrm{SD} 10 \mathrm{yr} 4 \mathrm{~m})\end{array}$ & $\begin{array}{l}20 \mathrm{yr} 1 \mathrm{~m} \\
(\mathrm{SD} 4 \mathrm{yr} 8 \mathrm{~m})\end{array}$ & $\begin{array}{l}16 \mathrm{yr} 10 \mathrm{~m} \\
(\mathrm{SD} 3 \mathrm{yr} 7 \mathrm{~m})\end{array}$ & $\begin{array}{l}18 \text { yr } 6 \mathrm{~m} \\
(\mathrm{SD} 8 \mathrm{yr} 7 \mathrm{~m})\end{array}$ & NSig \\
\hline $\begin{array}{l}\text { Preoperative partial seizure } \\
\text { frequency (per month) (PSF) }\end{array}$ & $\begin{array}{l}31 \cdot 8 \\
(\text { SD } 68 \cdot 17) \\
\text { (range 4-200) }\end{array}$ & $\begin{array}{l}14 \cdot 0 \\
(\text { SD } 10 \cdot 56) \\
\text { (range } 8-32)\end{array}$ & $\begin{array}{l}18 \cdot 0 \\
(\text { SD 17.41) } \\
\text { (range 15-60) }\end{array}$ & $\begin{array}{l}26 \cdot 6 \\
(\text { SD } 36 \cdot 35) \\
\text { (range } 4-100)\end{array}$ & NSig \\
\hline $\begin{array}{l}\text { Postoperative seizure total } \\
\text { before repeat Neuropsychological } \\
\text { assessment (PST) }\end{array}$ & $\begin{array}{l}8 \cdot 55 \\
(\mathrm{SD} 19 \cdot 24) \\
\text { (range } 0-60)\end{array}$ & $\begin{array}{l}1.5 \\
(\text { SD } 1 \cdot 69) \\
\text { (range } 0-4)\end{array}$ & $\begin{array}{l}1 \cdot 11 \\
(\text { SD } 3 \cdot 33) \\
\text { (range } 0-10)\end{array}$ & $\begin{array}{l}1 \cdot 071 \\
(\text { SD } 2 \cdot 16) \\
\text { (range } 0-6)\end{array}$ & NSig \\
\hline Neuropathology & $\begin{array}{l}2 \text { MTS } \\
2 \text { Tumour } \\
1 \text { CD } \\
5 \text { NS } \\
1 \text { hamartoma }\end{array}$ & $\begin{array}{l}5 \text { MTS } \\
1 \text { Tumour } \\
1 \text { NS } \\
1 \text { hamartoma }\end{array}$ & $\begin{array}{l}8 \text { MTS } \\
1 \text { NS }\end{array}$ & $\begin{array}{l}12 \text { MTS } \\
2 \text { NS }\end{array}$ & \\
\hline
\end{tabular}

Key: RTL right temporal lobectomy; RAH right amygdalo-hippocampectomy; LTL left temporal lobectomy; LAH left amygdalohippocampectomy; MTS mesial temporal sclerosis; CD cortical dysplasia; NS non-specific pathology; NSig non-significant.

sleep EEG. Where the results of any of these investigations suggested the possibility of bilateral temporal lobe damage, they then had an intracarotid Sodium Amytal (WADA) test (to determine the likelihood of surgery producing unacceptable memory deficits) and the implantation of foramen ovale electrodes with video telemetry to further determine the side of onset of seizures. Where foramen ovale electrode implantation did not provide conclusive lateralisation and localisation of the epileptic focus, depth electrode implantation was carried out. Patients were selected for operation on the basis of a combination of the following: a) Those with a structural lesion shown to be outside mesial temporal lobe structures were usually selected for temporal lobectomy; b) Patients in whom the onset of seizures was seen to be in mesial temporal structures by foramen ovale/depth electrode recordings and in whom the unoperated temporal lobe would not be able to support memory on the basis of the Sodium Amytal test, were normally offered an amygdalohippocampectomy; c) In doubtful cases, the decision was made at operation on the basis of the electrocorticography pattern. ${ }^{18}$

Pre- (and post) operative neuropsychological assessment included the following measures: a) A short form of the WAIS-R ${ }^{19}$ using the Vocabulary Comprehension, Similarities, Block Design and Object Assembly subtests. Prorated Verbal and Performance IQ values, as well as "General Ability IQ" and "VerbalSpatial Contrast IQ" ${ }^{20}$ were computed. The last two measures are derived from a principal components analysis of the WAIS-R. "General Ability", a single component, accounts for most of the variance in WAIS-R subtest scores. Derived IQ values have a mean (SD) of 100 (15). "Verbal-Spatial Contrast" a second component, contrasts verbal and performance subtest scores; scores above 100 indicate relative superiority of spatial over verbal abilities, the converse applying to scores below $100 ; \mathrm{b}$ ) The Logical Memory (LM) passages (immediate and one hour delayed recall) and the Verbal
Paired Associates test from the Wechsler Memory Scale. ${ }^{21}$ Total ideas recalled for the LM passages were summed across the two stories. Delayed recall was also expressed as a percentage of immediate recall; ${ }^{22}$ c) Immediate recall of visuospatial material (the Benton Visual Retention Test : BVRT ${ }^{23} 10 \mathrm{~s}$ stimulus presentation) and delayed recall (45 minutes) of the Rey Ostereith Complex figure. The latter provided a measure of percentage recall; ${ }^{22}$ d) Digit Span (from the WAIS-R) and Spatial Span using the Corsi block tapping test. ${ }^{24}$

The neuropsychological assessment and routine EEGs were repeated between one and four months post-operatively. Parallel forms of the learning and memory tests were used.

\section{Statistical analysis}

Analyses of variance (ANOVAs) and of covariance (ANCOVAs) were carried out using SPSS-PC ${ }^{+25}$ Subjects' data were classified according to side (right or left) and type (AH or TL) of operation. ANCOVAs incorporated epilepsy-related variables (age at surgery (AS), age at onset of seizures (AOS), age at onset of chronic seizures (AOCS), time between onset of chronic seizures and surgery (TCS), and pre- and post-operative seizure frequency/ total) as covariates. Pre-operative seizure frequency (PSF) was covaried with pre-operative cognitive measures and post-operative seizure total (PST) with post-operative and change measures. The extent of change in scores resulting from the operation was computed by subtracting the post-operative scores from preoperative values. The significance of results was assessed for $p<0 \cdot 05$. Pearson ProductMoment correlations were also assessed at this level.

As the data are preliminary, no correction was applied for multiple tests.

\section{Results}

\section{1) Patient characteristics}

The patient groups did not differ on mean age at surgery. Mean age at first seizure and age at 
Table 2 Pre-and postoperative mean (SD) values of verbal IQ (VIQ), Performance IQ (PIQ), General Ability and Verbal-Spatial Contrast IQs and differences in mean values $(p<0.05)$

\begin{tabular}{|c|c|c|c|c|c|}
\hline & $R T L$ & $L T L$ & $R A H$ & $L A H$ & Difference \\
\hline $\begin{array}{l}\text { Preoperative } \\
\text { VIQ }\end{array}$ & $\begin{array}{r}92.55 \\
(12.98)\end{array}$ & $\begin{array}{c}88 \cdot 50 \\
(12 \cdot 42)\end{array}$ & $\begin{array}{c}88 \cdot 11 \\
(17 \cdot 49)\end{array}$ & $\begin{array}{c}89 \cdot 29 \\
(11 \cdot 45)\end{array}$ & NSig \\
\hline $\begin{array}{l}\text { Postoperative } \\
\text { VIQ }\end{array}$ & $\begin{array}{c}94 \cdot 09 \\
(13 \cdot 66)\end{array}$ & $\begin{array}{c}86 \cdot 25 \\
(14 \cdot 49)\end{array}$ & $\begin{array}{c}89 \cdot 33 \\
(19 \cdot 15)\end{array}$ & $\begin{array}{c}87 \cdot 57 \\
(11 \cdot 38)\end{array}$ & NSig \\
\hline $\begin{array}{l}\text { Preoperative } \\
\text { PIQ }\end{array}$ & $\begin{array}{c}95.91 \\
(13.49)\end{array}$ & $\begin{array}{c}90 \cdot 00 \\
(14 \cdot 32)\end{array}$ & $\begin{array}{c}97 \cdot 56 \\
(20.92)\end{array}$ & $\begin{array}{c}97 \cdot 86 \\
(20 \cdot 36)\end{array}$ & NSig \\
\hline $\begin{array}{l}\text { Postoperative } \\
\text { PIQ }\end{array}$ & $\begin{array}{c}98.91 \\
(15 \cdot 85)\end{array}$ & $\begin{array}{c}98 \cdot 38 \\
(13 \cdot 50)\end{array}$ & $\begin{array}{c}96 \cdot 11 \\
(17 \cdot 93)\end{array}$ & $\begin{array}{c}96 \cdot 14 \\
(15 \cdot 87)\end{array}$ & NSig \\
\hline $\begin{array}{l}\text { Preoperative General } \\
\text { Ability IQ }\end{array}$ & $\begin{array}{l}92 \cdot 27 \\
(9 \cdot 36)\end{array}$ & $\begin{array}{c}89 \cdot 38 \\
(12 \cdot 26)\end{array}$ & $\begin{array}{c}92 \cdot 63 \\
(14 \cdot 67)\end{array}$ & $\begin{array}{l}91.07 \\
(9.01)\end{array}$ & NSig \\
\hline $\begin{array}{l}\text { Postoperative General } \\
\text { Ability IQ }\end{array}$ & $\begin{array}{c}94 \cdot 55 \\
(12 \cdot 36)\end{array}$ & $\begin{array}{l}90 \cdot 38 \\
(9 \cdot 79)\end{array}$ & $\begin{array}{c}93 \cdot 63 \\
(15 \cdot 16)\end{array}$ & $\begin{array}{c}90 \cdot 14 \\
(11 \cdot 04)\end{array}$ & NSig \\
\hline $\begin{array}{l}\text { Preoperative Verbal-Spatial } \\
\text { Contrast IQ }\end{array}$ & $\begin{array}{l}103 \cdot 91 \\
(12 \cdot 64)\end{array}$ & $\begin{array}{c}99 \cdot 63 \\
(11 \cdot 19)\end{array}$ & $\begin{array}{l}108 \cdot 00 \\
(21 \cdot 35)\end{array}$ & $\begin{array}{l}105 \cdot 71 \\
(15 \cdot 56)\end{array}$ & NSig \\
\hline $\begin{array}{l}\text { Postoperative Verbal-Spatial } \\
\text { Contrast IQ }\end{array}$ & $\begin{array}{l}101 \cdot 82 \\
(14 \cdot 58)\end{array}$ & $\begin{array}{l}109 \cdot 25 \\
(12 \cdot 99)\end{array}$ & $\begin{array}{l}107 \cdot 13 \\
(17 \cdot 80)\end{array}$ & $\begin{array}{l}108 \cdot 86 \\
(14 \cdot 05)\end{array}$ & NSig \\
\hline
\end{tabular}

onset of chronic seizures was less for those having left-sided operations than for those having right-sided surgery (AOS; $F(1,38)=$ 5.51; AOCS F $(1,38)=4.69 p<0.05$ in each case) but there was no significant difference for the type of operation received. There was neither a main effect of SIDE nor OP when considering TCS, PSF and PST.

\section{2) Intelligence}

Pre- and postoperative measures of Verbal and Performance IQ (VIQ and PIQ), General Ability IQ and Verbal-Spatial Contrast IQ are given in table 2 .

Preoperative mean scores for VIQ and PIQ were all within one standard deviation of the mean. No differences between groups were revealed by ANOVA for either measure although the LTLs tended to score lower than the RTLs on pre- and postoperative VIQ. No differences between groups were established when the epilepsy variables were included in the analyses. No differences between groups were found for postoperative VIQ and PIQ, General Ability and Verbal-Spatial contrast IQ measures.

There were no differences between groups on mean change scores for VIQ. For PIQ, AH resulted in mild deterioration, whereas TL was followed by an improvement $[\mathrm{F}(1,36)=4.72$, $\mathrm{p}<0.05]$. This finding also held when the

Table 3 Pre- and postoperative mean (SD) immediate, delayed and percentage recall of the Logical Memory Passages and learning score on Verbal Paired Associates and differences in mean values $(p<0.05)$

\begin{tabular}{|c|c|c|c|c|c|}
\hline & $R T L$ & $L T L$ & $R A H$ & $L A H$ & Difference \\
\hline $\begin{array}{l}\text { Preoperative LM } \\
\text { Immediate Recall }\end{array}$ & $\begin{array}{c}19 \cdot 91 \\
(8 \cdot 48)\end{array}$ & $\begin{array}{l}16 \cdot 44 \\
(7 \cdot 31)\end{array}$ & $\begin{array}{c}13 \cdot 28 \\
(7 \cdot 79)\end{array}$ & $\begin{array}{c}19 \cdot 50 \\
(5 \cdot 42)\end{array}$ & $\begin{array}{l}\text { SIDE } \times \text { OP } \\
p=0.068 \\
(A N O V A)\end{array}$ \\
\hline $\begin{array}{l}\text { Postoperative LM } \\
\text { Immediate Recall }\end{array}$ & $\begin{array}{l}18 \cdot 55 \\
(9 \cdot 01)\end{array}$ & $\begin{array}{l}12 \cdot 13 \\
(6 \cdot 31)\end{array}$ & $\begin{array}{c}15 \cdot 72 \\
(9 \cdot 47)\end{array}$ & $\begin{array}{c}14 \cdot 29 \\
(5 \cdot 17)\end{array}$ & NSig \\
\hline $\begin{array}{l}\text { Preoperative LM } \\
\text { Delayed Recall }\end{array}$ & $\begin{array}{l}15 \cdot 45 \\
(6 \cdot 49)\end{array}$ & $\begin{array}{l}12 \cdot 25 \\
(8 \cdot 04)\end{array}$ & $\begin{array}{l}9 \cdot 06 \\
(8 \cdot 27)\end{array}$ & $\begin{array}{c}13 \cdot 27 \\
(7 \cdot 34)\end{array}$ & NSig \\
\hline $\begin{array}{l}\text { Postoperative LM } \\
\text { Delayed Recall }\end{array}$ & $\begin{array}{l}15 \cdot 68 \\
(7 \cdot 74)\end{array}$ & $\begin{array}{l}9 \cdot 00 \\
(7 \cdot 04)\end{array}$ & $\begin{array}{l}11 \cdot 16 \\
(9 \cdot 38)\end{array}$ & $\begin{array}{l}9 \cdot 14 \\
(5 \cdot 51)\end{array}$ & NSig \\
\hline $\begin{array}{l}\text { Preoperative LM } \\
\text { Percent recall }\end{array}$ & $\begin{array}{l}79 \cdot 00 \\
(10 \cdot 22)\end{array}$ & $\begin{array}{l}69 \cdot 63 \\
(22 \cdot 04)\end{array}$ & $\begin{array}{l}54 \cdot 89 \\
(28 \cdot 67)\end{array}$ & $\begin{array}{l}83 \cdot 92 \\
(62 \cdot 57)\end{array}$ & NSig \\
\hline $\begin{array}{l}\text { Postoperative LM } \\
\text { Percent recall }\end{array}$ & $\begin{array}{l}80 \cdot 64 \\
(15 \cdot 02)\end{array}$ & $\begin{array}{l}59 \cdot 25 \\
(22 \cdot 56)\end{array}$ & $\begin{array}{l}66 \cdot 11 \\
(27 \cdot 03)\end{array}$ & $\begin{array}{l}59 \cdot 57 \\
(26 \cdot 45)\end{array}$ & NSig \\
\hline $\begin{array}{l}\text { Preoperative Paired } \\
\text { Associate Learning }(\max 21)\end{array}$ & $\begin{array}{l}12 \cdot 45 \\
(3 \cdot 08)\end{array}$ & $\begin{array}{c}13 \cdot 75 \\
(2 \cdot 43)\end{array}$ & $\begin{array}{l}12 \cdot 00 \\
(4 \cdot 30)\end{array}$ & $\begin{array}{l}14 \cdot 61 \\
(3 \cdot 49)\end{array}$ & $\begin{array}{l}\text { SIDE }(\mathrm{L}>\mathrm{R}) \\
(\text { ANCOVA) }\end{array}$ \\
\hline $\begin{array}{l}\text { Postoperative Paired } \\
\text { Associate Learning }(\max 21)\end{array}$ & $\begin{array}{l}14 \cdot 86 \\
(2 \cdot 26)\end{array}$ & $\begin{array}{l}8 \cdot 77 \\
(4 \cdot 17)\end{array}$ & $\begin{array}{l}12 \cdot 81 \\
(3 \cdot 92)\end{array}$ & $\begin{array}{l}12 \cdot 61 \\
(4 \cdot 49)\end{array}$ & $\begin{array}{l}\text { SIDE }(L<R) \\
\text { and SIDE } \times \text { OP }\end{array}$ \\
\hline
\end{tabular}

epilepsy variables were included as covariates. No differences between groups were seen.on change scores for the General Ability IQ measure, but for the Verbal-Spatial Contrast IQ measure, a SIDE effect was found; rightsided surgery produced a lower score (reflecting a relative deterioration of visuospatial with respect to verbal ability), and left-sided surgery produced an increased score $[F(1,36)=4 \cdot 33$, $\mathrm{p}<0.05]$. This finding was upheld when patient epilepsy-related variables were included as covariates.

Thus pre- and postoperatively absolute VIQ and PIQ measures did not distinguish between the TL and AH operations. Change scores on PIQ did differentiate between type of operation, with the $\mathrm{AH}$ leading to a mild deterioration and TL to improvement.

\section{3) Memory}

\section{A) Verbal material}

Pre- and postoperative scores on immediate delayed and percentage recall of the LM passages, and verbal paired associate learning are given in table 3.

Preoperatively a SIDE $x$ OP interaction approached significance for mean scores on the immediate $\mathrm{LM}$ recall $[\mathrm{F}(1,36)=3.55, \mathrm{p}=$ 0.068 ] and when the epilepsy variables were included in the ANCOVAs. Thus RAHs tended to score less well than RTLs, but LAHs tended to score better than LTLs on this measure. No main effects or interactions were found for preoperative delayed or percentage recall. For preoperative verbal paired associate learning, the SIDE effect approached significance $[F(1,36)=3 \cdot 25, p=0.08]$. This reached significance when AOS, AOCS, TCS were included as covariates $(p<0.05$ in each case). Thus surprisingly, patients receiving leftsided operations scored better on this test of verbal learning before surgery than the rightsided cases.

Postoperatively no significant main effects or interactions were found for immediate, delayed or percentage recall of the LM passages, despite the tendency for those receiving leftsided surgery to perform worse than rightsided cases on these tests. For verbal paired associate learning, a main effect of SIDE [F $(1,36)=5.37, p<0.05]$ and a SIDE $\times$ OP interaction $[F(1,36)=5.52, p<0.05]$ were obtained. These were also found when AS, TCS and PST were included as covariates; only the SIDE effect remained when AOS and AOCS were covaried.

For change scores, an effect of SIDE was found for immediate story recall $[F(1,36)=$ 5.37, $\mathrm{p}<0.05$ ]; left-sided cases showed clear deterioration as a result of surgery. ANCOVAs revealed a similar pattern of results. Delayed recall change scores yielded a main effect of SIDE when AOS, AOCS, TCS and PST were considered ( $p<0.05$ in each case). No significant main effects or interactions were found for percentage recall change scores. Change scores on verbal paired associate learning yielded a significant effect of SIDE [F $(1,36), 18.67, \mathrm{p}<0.001]$ and the SIDE $\times$ OP effect approached significance $[F(1,36)=$ 
Table 4 Pre-and postoperative mean (SD) correct and error scores on the Benton Visual Retention Test (BVRT) and percentage recall of the Rey figure and differences in mean values $(p<0.05)$

\begin{tabular}{|c|c|c|c|c|c|}
\hline & $R T L$ & $L T L$ & $R A H$ & $L A H$ & Difference \\
\hline $\begin{array}{l}\text { BVRT Preoperative } \\
\text { Correct }\end{array}$ & $\begin{array}{l}6 \cdot 91 \\
(2 \cdot 11)\end{array}$ & $\begin{array}{l}6 \cdot 25 \\
(1 \cdot 17)\end{array}$ & $\begin{array}{l}4 \cdot 89 \\
(1 \cdot 76)\end{array}$ & $\begin{array}{l}6 \cdot 86 \\
(1 \cdot 35)\end{array}$ & SIDE $\times$ OP \\
\hline $\begin{array}{l}\text { BVRT Postoperative } \\
\text { Correct }\end{array}$ & $\begin{array}{l}5 \cdot 64 \\
(1 \cdot 50)\end{array}$ & $\begin{array}{l}6 \cdot 25 \\
(1 \cdot 67)\end{array}$ & $\begin{array}{l}4 \cdot 67 \\
(2 \cdot 00)\end{array}$ & $\begin{array}{l}5 \cdot 78 \\
(1 \cdot 31)\end{array}$ & NSig \\
\hline $\begin{array}{l}\text { BVRT Preoperative } \\
\text { Errors }\end{array}$ & $\begin{array}{l}4 \cdot 64 \\
(3 \cdot 61)\end{array}$ & $\begin{array}{l}5 \cdot 00 \\
(1 \cdot 93)\end{array}$ & $\begin{array}{l}7 \cdot 67 \\
(3 \cdot 20)\end{array}$ & $\begin{array}{l}4 \cdot 79 \\
(2 \cdot 49)\end{array}$ & $\begin{array}{l}\text { SIDE } \times \text { OP } \\
0 \cdot 084 \\
(A N O V A) \\
\text { SIDE } \times \text { OP } \\
\text { OP }(R>\text { L) (ANCOVA) }\end{array}$ \\
\hline $\begin{array}{l}\text { BVRT Preoperative } \\
\text { Errors }\end{array}$ & $\begin{array}{l}6 \cdot 09 \\
(2 \cdot 34)\end{array}$ & $\begin{array}{l}4 \cdot 63 \\
(2 \cdot 88)\end{array}$ & $\begin{array}{l}8 \cdot 22 \\
(4 \cdot 74)\end{array}$ & $\begin{array}{l}5 \cdot 79 \\
(2 \cdot 15)\end{array}$ & $\begin{array}{l}\operatorname{SIDE} \\
(\mathrm{R}>\mathrm{L})\end{array}$ \\
\hline $\begin{array}{l}\text { Preoperative Rey } \\
\text { Percentage Recall }\end{array}$ & $\begin{array}{l}54 \cdot 09 \\
(14 \cdot 47)\end{array}$ & $\begin{array}{l}50 \cdot 88 \\
(13 \cdot 35)\end{array}$ & $\begin{array}{l}47 \cdot 11 \\
(18 \cdot 25)\end{array}$ & $\begin{array}{l}57 \cdot 14 \\
(17 \cdot 77)\end{array}$ & NSig \\
\hline $\begin{array}{l}\text { Postoperative Rey } \\
\text { Percentage Recall }\end{array}$ & $\begin{array}{l}53 \cdot 55 \\
(11 \cdot 12)\end{array}$ & $\begin{array}{l}51 \cdot 25 \\
(9 \cdot 48)\end{array}$ & $\begin{array}{l}46 \cdot 11 \\
(21 \cdot 97)\end{array}$ & $\begin{array}{l}59 \cdot 50 \\
(10 \cdot 79)\end{array}$ & NSig \\
\hline
\end{tabular}

$3.37, \mathrm{p}<0.06]$. Thus left-sided surgery was accompanied by deterioration on this test, and this held when the epilepsy variables were included as covariates; the SIDE $\times$ OP interaction reached significance for the covariate of TCS.

\section{B) Visuospatial material}

Two scores were derived from the BVRT, the number of correct figure reproductions (maximum 10) and the number of errors. Pre- and postoperative correct and error scores and preand postoperative recall of the Rey figure are shown in table 4.

Preoperatively a significant SIDE $\times$ OP interaction was obtained for the Benton correct scores $[F(1,38)=6.39, p<0.05]$ and the same interaction approached significance for the error score $[F(1,38)=3 \cdot 15, p=0.084]$. When AS was included as a covariate, the OP effect approached significance $[F(1,38)=$ $8.76, p=0.057]$ and the interaction became significant $[F(1,38)=7.67, p<0.01]$. The interaction and/or the OP effect reached significance when the epilepsy variables were included as covariates. No significant main effects or interactions were obtained for the pre-operative Rey figure percentage recall measure.

Postoperatively no main effects or interactions were significant for the BVRT correct scores. Analysis of error scores yielded a main

Table 5 Pre-and postoperative mean (SD) Digit Spans (forwards and backwards) and Spatial Spans (forwards and backwards) and differences in mean values $(p<0.05)$

\begin{tabular}{|c|c|c|c|c|c|}
\hline & $R T L$ & $L T L$ & $R A H$ & $L A H$ & Difference \\
\hline $\begin{array}{l}\text { Digit Span } \\
\text { Forwards Preoperative }\end{array}$ & $\begin{array}{l}6 \cdot 64 \\
(1 \cdot 21)\end{array}$ & $\begin{array}{l}6.13 \\
(0.99)\end{array}$ & $\begin{array}{l}6 \cdot 22 \\
(1 \cdot 09)\end{array}$ & $\begin{array}{l}6 \cdot 79 \\
(1 \cdot 31)\end{array}$ & NSig \\
\hline $\begin{array}{l}\text { Digit Span } \\
\text { Forwards Postoperative }\end{array}$ & $\begin{array}{l}5 \cdot 90 \\
(1 \cdot 10)\end{array}$ & $\begin{array}{l}6 \cdot 00 \\
(0 \cdot 76)\end{array}$ & $\begin{array}{l}5 \cdot 78 \\
(1 \cdot 20)\end{array}$ & $\begin{array}{l}6 \cdot 71 \\
(1 \cdot 64)\end{array}$ & $\begin{array}{l}\text { SIDE } \\
\text { (L }>\text { R) } \\
\text { (ANCOVA) }\end{array}$ \\
\hline $\begin{array}{l}\text { Digit Span } \\
\text { Backwards Preoperative }\end{array}$ & $\begin{array}{l}4 \cdot 09 \\
(1 \cdot 30)\end{array}$ & $\begin{array}{l}4 \cdot 75 \\
(0 \cdot 89)\end{array}$ & $\begin{array}{l}4 \cdot 44 \\
(1 \cdot 94)\end{array}$ & $\begin{array}{l}4 \cdot 93 \\
(1 \cdot 33)\end{array}$ & $\begin{array}{l}\text { SIDE } \\
\text { (L > R) } \\
\text { (ANCOVA) }\end{array}$ \\
\hline $\begin{array}{l}\text { Digit Span } \\
\text { Backwards Postoperative }\end{array}$ & $\begin{array}{l}4 \cdot 40 \\
(0 \cdot 97)\end{array}$ & $\begin{array}{l}4 \cdot 75 \\
(1 \cdot 17)\end{array}$ & $\begin{array}{l}4 \cdot 44 \\
(1 \cdot 42)\end{array}$ & $\begin{array}{l}5 \cdot 79 \\
(1 \cdot 25)\end{array}$ & $\begin{array}{l}\text { SIDE } \\
(\mathrm{L}>\mathrm{R})\end{array}$ \\
\hline $\begin{array}{l}\text { Spatial Span } \\
\text { Forwards Preoperative }\end{array}$ & $\begin{array}{l}5 \cdot 27 \\
(1 \cdot 49)\end{array}$ & $\begin{array}{l}5 \cdot 63 \\
(0 \cdot 92)\end{array}$ & $\begin{array}{l}5 \cdot 13 \\
(0 \cdot 83)\end{array}$ & $\begin{array}{l}5 \cdot 79 \\
(1 \cdot 12)\end{array}$ & NSig \\
\hline $\begin{array}{l}\text { Spatial Span } \\
\text { Forwards Postoperative }\end{array}$ & $\begin{array}{l}5 \cdot 00 \\
(1.09)\end{array}$ & $\begin{array}{l}5 \cdot 50 \\
(1 \cdot 51)\end{array}$ & $\begin{array}{l}4 \cdot 89 \\
(1 \cdot 05)\end{array}$ & $\begin{array}{l}5 \cdot 36 \\
(0 \cdot 74)\end{array}$ & NSig \\
\hline $\begin{array}{l}\text { Spatial Span } \\
\text { Backwards Preoperative }\end{array}$ & $\begin{array}{l}5 \cdot 18 \\
(1 \cdot 66)\end{array}$ & $\begin{array}{l}5 \cdot 38 \\
(1 \cdot 06)\end{array}$ & $\begin{array}{l}5 \cdot 29 \\
(0 \cdot 76)\end{array}$ & $\begin{array}{l}4.93 \\
(0 \cdot 99)\end{array}$ & NSig \\
\hline $\begin{array}{l}\text { Spatial Span } \\
\text { Backwards Postoperative }\end{array}$ & $\begin{array}{l}5 \cdot 09 \\
(1 \cdot 04)\end{array}$ & $\begin{array}{l}5 \cdot 50 \\
(1 \cdot 19)\end{array}$ & $\begin{array}{l}4 \cdot 50 \\
(1 \cdot 31)\end{array}$ & $\begin{array}{l}5 \cdot 36 \\
(0 \cdot 93)\end{array}$ & NSig \\
\hline
\end{tabular}

effect of SIDE $[F(1,38)=4.32, p<0.05]$ with right-sided surgery resulting in a greater number of errors than left-sided surgery. This held when AS, TCS, and PST were included as covariates. As with pre-operative scores, no significant main effects or interactions were obtained for the Rey percentage recall scores.

BVRT correct change scores showed a significant SIDE $\times$ OP interaction $[F(1 \cdot 36)=$ $4.98, p<0.05$ ] with RTLS showing more deterioration than RAHs, and LTLs showing less deterioration than LAHs across the operation. This interaction held for all epilepsy variable covariates.

Error change scores yielded no main effects. The SIDE $\times$ OP interaction approached significance when TCS was included as a covariate. Thus RTLs tended to show greater deterioration than RAHs, and LAHs tended to show greater deterioration than LTLs on this measure.

No significant differences or interactions were found for the Rey change scores.

\section{C) Digit and spatial spans}

Mean digit and spatial spans (forwards and backwards) pre- and postoperatively are shown in table 5.

Preoperatively no main effects or interactions were detected for digit span forwards (DSF). This was also true for digit span backwards (DSB) except when AS was included as a covariate, when a main effect of SIDE was obtained $[F(1,34)=4.69, p<$ $0 \cdot 05]$. No main effects or interactions were revealed for spatial span forwards (SSF) or backwards (SSB).

Postoperatively DSF scores yielded a significant effect of side of operation with postoperative seizure frequency as a covariate $[F$ $(1,36)=5 \cdot 77, p<0.05]$. DSB yielded a main effect of SIDE $[F(1,36)=5.32, p<0.05]$ and for both DSF and DSB, right-sided cases scored less well than left-operated patients. The DSB SIDE effect held with AS and TCS covariates, and approached significance with other epilepsy-related covariates. No main effects or interactions were found for postoperative measures of SSF or SSB.

Change scores for the span measures yielded only SIDE effects for DSF, when AS and PST were covaried, with the right-sided cases showing greater deterioration than the left-sided cases. No significant differences between the groups' mean change scores were, found for DSB, SFF or SFB measures.

\section{Relationship between epilepsy variables and} cognitive performance

For each of the four operated groups, correlations were computed between the cognitive scores (pre- and postoperative) and in turn AOS, AOCS, TCS and AS. Correlations were undertaken between PSF and preoperative scores, and between PST and postoperative scores on cognitive tests. In view of the small numbers in each group, results can provide only initial impressions concerning the relationships between these variables and cognitive measures. 
Different patterns of correlations emerged for those undergoing left- or right-sided surgery. Thus for right-sided cases, AOS or AOCS were related to some index of verbal learning or memory: (RAHs: postoperative delayed LM recall, postoperative verbal paired associative learning $\mathrm{p}<0.01$; RTLs: postoperative percentage LM recall). Thus later epilepsy onset was related to better performance on these verbal tests. Age at surgery was negatively correlated with postoperative General Ability IQ (RAHs). This probably reflects a change in the relative superiority of visuospatial over verbal skills since for RAHs, younger age at surgery was associated with a greater superiority of visuospatial over verbal skills than was an older age at surgery. Later surgery for the RTLs was associated with poor postoperative recall of the Rey figure whereas those RAHs who were older at surgery had better preoperative recall of the figure than those who were younger at the time of operation.

For left-sided cases, no verbal learning or memory measures correlated with epilepsyrelated variables. Instead BVRT correct and error scores seemed more sensitive to the effects of prolonged seizure activity: AOCS correlated negatively with postoperative BVRT errors for the LTLs and with preoperative error scores for the LAHs, and positively with BVRT preoperative correct scores for the LAHs. Preoperative BVRT correct and error scores, correlated with TCS negatively and positively respectively for the LAHS. Poor postoperative seizure control in the LTLs was associated with poorer recall of verbal (immediate LM recall, paired associate learning) and some visuospatial material (percentage recall of the Rey figure), suggesting that for these patients at least, ongoing seizure activity was disrupting information storage.

\section{Discussion}

This study documents pre- and short term postoperative cognitive functioning, and change in cognitive performance in 42 adults who had either a standard temporal lobectomy or a selective amygdalo-hippocampectomy for the relief of chronic temporal lobe epilepsy, and considered whether $\mathrm{AH}$ produces less cognitive impairment than TL. As yet numbers for each of the four operations are relatively small so the current findings are preliminary.

Although no differences between groups were found on pre- or postoperative IQ measures, change scores indicated that while TL (irrespective of side) was followed by an improvement in PIQ, AH was followed by mild deterioration. Right-sided surgery was followed by a relative lessening of the superiority of visuospatial over verbal skills, while the converse was true for left-sided operations. This is consistent with predictions based on side of surgery.

As found by Powell et al, ${ }^{12}$ immediate recall of the LM stories was in general a more sensitive measure than delayed or percentage recall. Although Powell et al had found a significant postoperative effect of side of TL on this measure, only change scores resulting from the operation revealed an effect of side here, and also for delayed recall. For immediate recall, left-sided surgery resulted in deterioration; RTL also led to deterioration (but to a lesser degree) whereas RAH produced a mild degree of improvement. For delayed recall both left-sided groups' scores fell, whereas both right-sided groups (AH more than TL) showed some improvement. Thus on these measures there is only minimal evidence that $\mathrm{AH}$ and TL have different outcomes, and then only for the right-sided cases.

For verbal paired association, not reported earlier, ${ }^{12}$ there was a clear postoperative effect of side of lesion and an interaction between side and type of surgery. Thus RTLs performed better than RAHs, but LTLs performed worse than LAHs. Left-sided surgery produced clear deterioration on this test (LTLs showing more deterioration than LAHs) and right-sided surgery produced some improvement (RAHs showing less improvement than RTLs). The SIDE $\times$ OP interaction approached or reached significance depending upon the covariates. Thus on this measure there is some indication that LAH will produce marginally less cognitive impairment than LTL, but that RAH will result in less improvement than RTL.

As reported earlier, ${ }^{12}$ delayed recall of the Rey Complex figure did not distinguish between right- or left-sided surgery and there was no differential effect of the two types of operation. The BVRT scores were more sensitive. For "correct" change scores, the RTLs showed more deterioration than the RAHs, but the LTLs showed less deterioration than LAHs, suggesting marginal superiority of RAH over RTL on this measure. This interaction approached significance for error scores. Postoperatively only a significant effect of side of operation was found, and only for error scores, with right-sided cases making more errors than left-sided cases.

No differential effect of type of operation (AH or TL) was found for the span measures. A significant postoperative effect of side of surgery was found for DSF and DSB, and right-sided cases showed more deterioration across the operation on DSF than did those with left-sided surgery.

The observation of SIDE $\times$ OP interactions (either reaching or approaching statistical significance) for preoperative measures, suggests that some patients' characteristics may be affecting cognitive performance. It is conceivable that underlying neuropathology may relate to performance on the memory tests. As can be seen from table 1, unlike the TL cases, most of the AH cases were found to show mesial temporal sclerosis (MTS). McMillan et $a l^{13}$ noted that preoperative differences in verbal memory in MTS patients were unremarkable for side of lesion, and the overall level of verbal recall fell between that of the rightand left-sided groups with tumour-like pathology, with the right-sided cases showing above average and the left-sided cases below average verbal memory. Although the present numbers 
are small it is possible that the SIDE $\times$ OP interactions could be accounted for by the tumour-like pathology cases in the TL groups (in addition to the MTS cases) and the absence of such tumour-like pathology cases in the $\mathrm{AH}$ groups.

As with Powell et al's study ${ }^{12}$ AOCS again predicted cognitive scores more consistently for right-sided than left-sided cases. Age at surgery showed a stronger association with cognitive performance for the right-sided than for the left-sided cases, as was previously reported. In our study right-sided cases with later onset seizure activity developed better verbal learning and memory suggesting that it is not only verbal intellectual abilities ${ }^{12}$ that can develop better in the absence of early rightsided seizures. Similarly, considering BVRT scores, some aspects of non verbal activities develop better in the absence of early left-sided seizures. These findings are relevant when assessing patients for epilepsy surgery since early onset epilepsy may be expected to influence the likelihood of finding impairment in both verbal and non-verbal memory skills.

Postoperative seizure total related to few cognitive measures. For the RTLs and LTLs poor seizure control affected performance on material specific tests related to the side of lesion (although a more generalised effect on cognitive performance was found for the LTLs). The RAHs and LAHs, overall showed little seizure activity before the postoperative assessment, thereby reducing the possibility of eliciting correlations.

In conclusion, there is some indication that on specific measures of cognitive functioning (verbal paired associate learning, immediate recall of visuospatial material) an $\mathrm{AH}$ rather than a TL performed on the temporal lobe thought to mediate that particular function will produce less pronounced impairment in terms of change resulting from operation, as indicated by short-term cognitive evaluation. Equally, however, an $\mathrm{AH}$ carried out on the temporal lobe not thought to mediate these skills appears to result in less improvement or more deterioration in that skill than will a TL, except in the case of delayed story recall, where $\mathrm{RAH}$ overall produced more improvement than RTL. Longer term outcome, for formal and everyday cognitive functioning and seizure control must, however, be evaluated.

1 Milner B. Disorders of learning and memory after temporal lobe lesions in man. Clin Neurosurg 1972;19:421-46.

2 Scoville WB, Milner B. Loss of recent memory after bilateral hippocampal lesions. 7 Neurol Neurosurg Psychiatry 1957;20:11-21.

3 Prisko L. Short-term memory in focal cerebral damage (unpublished PhD thesis). McGill University, 1963.

4 Kimura D. Right temporal-lobe damage: Perceptions of unfamiliar stimuli after damage. Arch Neurol 1963;8:264-71.

5 Milner B. Brain mechanisms suggested by studies of temporal lobes. In: Millikan CH, Darley FL, eds. Brain mechanisms underlying speech and language. New York: Grune and Stratton, 1967:122-32.

6 Milner B. Visual recognition and recall after right temporal lobe excision in man. Neuropsychologia 1968;6:191-209.

7 Corsi PM. Human memory and the medial temporal region of the brain (unpublished PhD thesis). McGill University, 1972.

8 Frisk V, Milner B. The relationship of working memory to the immediate recall of stories following unilateral temporal or frontal lobectomy. Neuropsychologia 1990: 28:121-35.

9 Goldstein LH, Canavan AGM, Polkey CE. Verbal and abstract designs paired associate learning after unilateral temporal lobectomy. Cortex 1988;24:41-52.

10 Goldstein LH, Canavan AGM, Polkey CE. Cognitive mapping after unilateral temporal lobectomy. Neuropsychologia 1989;27:167-77.

11 Jones-Gotman $\mathbf{M}$. Commentary: Testing hippocampal function. In: J Engel Jnr, ed. Surgical treatment of the epilepsies.
New York: Raven Press, 1987:203-11.

12 Powell GE, Polkey CE, McMillan TM. The new Maudsley series of temporal lobectomy. Short-term cognitive series of temporal lobectomy. Short-tern

13 McMillan TM, Powell GE, Janota I, Polkey CE. Relationship between neuropathology and cognitive functioning in temporal lobectomy patients. F Neurol Neurosurg Psychiatry 1987;50:167-76.

14 Polkey CE. Anterior temporal lobectomy at the Maudsley Hospital, London. In: J Engel Jnr, ed. Surgical treatment of the epilepsies. New York: Raven Press, 1987:641-5.

15 Wieser HG. Selective amygdalohippocampectomy: Indications, investigative technique and results. In: Symon $\mathrm{L}$, et al, eds. Advances and technical standards in neurosurgery $\mathrm{Vol}$ 13. Vienna: Springer-Verlag, 1986:39-133.

16 Wieser HG. Selective amygdalo-hippocampectomy for temporal lobe epilepsy. Epilepsia 1988;29 (Suppl 2):S100-13

17 Wieser HG, Yasargil MD. Selective amygdalohippocampectomy as a surgical treatment of mesiobasal limbic epilepsy. Surg Neurol 1982;17:445-57.

18 Polkey CE, Binnie CD, Janota I. Acute hippocampal recording and pathology at temporal lobe resection and recording and pathology at temporal lobe resection and

19 Wechsler D. The Wechsler Adult Intelligence Scale-Revised. New York: The Psychological Corporation, 1981.

20 Canavan AGM, Dunn G, McMillan TM. The principal components of the WAIS-R. Br $\boldsymbol{f}$ Clin Psychol components $1986 ; 25: 81-5$.

21 Wechsler D, Stone CP. The Wechsler Memory Scale. New York: Psychological Corporation, 1945.

22 Powell GE. The relationship between intelligence and verbal and spatial memory. $\mathcal{F}$ Clin Psychol 1979;35:335-40.

23 Benton AL. Revised Visual Retention Test Manual. New York: Psychological Corporation, 1974

24 Canavan AGM, Passingham RE, Marsden CD, Quinn N, Wyke M, Polkey CE. Sequencing ability in Parkinsonians, patients with frontal lobe lesions and patients who have undergone unilateral temporal lobectomies. Neuropsychologia 1989;27:787-98.

25 SPSS Inc. SPSS-PC+V2.0. Chicago, 1988 